\title{
Treatment of a Spinal Cord Hemitransection Injury with Keratin Biomaterial Hydrogel Elicits Recovery and Tissue Repair
}

\author{
Bailey V. Fearing, ${ }^{1,2}$ Christopher Hartley, ${ }^{3}$ Orrin Dayton, ${ }^{3}$ \\ Garrett Sherwood, ${ }^{3}$ Tamer AbouShwareb, ${ }^{1}$ and Mark E. Van Dyke ${ }^{4}$ \\ ${ }^{1}$ Wake Forest Institute for Regenerative Medicine, Winston Salem, NC 27157, USA \\ ${ }^{2}$ Wake Forest University Molecular Medicine and Translational Science Graduate Program, Winston Salem, NC 27157, USA \\ ${ }^{3}$ Department of Orthopaedic Surgery, Wake Forest University School of Medicine, Winston Salem, NC 27157, USA \\ ${ }^{4}$ School of Biomedical Engineering and Sciences, Virginia Polytechnic Institute and State University, Blacksburg, VA 24061, USA
}

Correspondence should be addressed to Mark E. Van Dyke; mvandyk5@vt.edu

Received 6 January 2014; Accepted 17 February 2014; Published 23 March 2014

Academic Editors: S. Lamponi and S. Liao

Copyright (C) 2014 Bailey V. Fearing et al. This is an open access article distributed under the Creative Commons Attribution License, which permits unrestricted use, distribution, and reproduction in any medium, provided the original work is properly cited.

\begin{abstract}
Medical care costs can reach an estimated value of $\$ 4$ billion for spinal cord injuries (SCI) each year in the USA alone. With no viable treatment options available, care remains palliative and aims to minimize lifelong disabilities and complications, such as immobility, bladder and bowel dysfunction, breathing problems, and blood clots. Human hair keratin biomaterials have demonstrated efficacy in peripheral nerve injury models and were shown to improve conduction delay and increase axon number and density. In this study, a keratin hydrogel was tested in a central nervous system (CNS) application of spinal cord hemisection injury. Keratin-treated rats showed increased survival rates as well as a better functional recovery of gait properties and bladder function. Histological results demonstrated reduced glial scar formation with keratin treatment and suggested a greater degree of beneficial remodeling and cellular influx. The data provided in this pilot study suggest the possibility of using a keratin-based treatment for SCI and warrant further investigation.
\end{abstract}

\section{Introduction}

More than 2.5 million people worldwide are effected by spinal cord injuries (SCI), with approximately 12,000 new cases each year in USA alone [1]. The majority of these cases are caused by motor vehicle accidents (36.5\%) and falls $(28.5 \%)$ and predominantly affect young males (80.7\%) [1]. Depending on the extent of the injury, survivors can experience severe lifelong disabilities, including immobility, pressure sores, autonomic dysreflexia, neurogenic pain, and breathing problems. Most SCIs will interrupt bladder and bowel functions because the nerves controlling these processes originate in the lower segments of the spinal cord and are subsequently cut off from brain input. In the case of the bladder, loss of neural input causes voiding to become abnormal and thus requires the chronic use of catheters. SCI injury has an even greater impact when lifetime costs are considered, which easily reach millions per patient. Medical care costs alone reach an estimated value of $\$ 4$ billion per year in USA [2]. Taking into account the fact that the majority of patients are young, active people who are now relegated to long-term palliative care, these numbers increase even more when considering loss of productivity and income wages [1].

Treatment options after SCI are limited, and life expectancies have not improved for patients since the 1980s. The use of the steroid methylprednisolone sodium succinate (MPSS) has fallen out of favor for many clinicians because of the lack of neurological improvements and serious side effects [3-5]. Spinal stabilization surgery is performed if the spinal cord is being compressed by a blood clot or herniated disc and is effective in terms of stabilizing the spine, relieving pressure, and preventing further deformities. However, it 
does nothing to address the damage that has already been done. Moreover, there is no current evidence to suggest that it offers advantages in functional outcomes [6, 7]. Physical therapy can offer some improvements, but the standard of care remains palliative.

After the initial injury, inflammation, cell death, and limited axon sprouting occur before a glial scar seals the site of injury. However, the lesion continues to expand due to secondary damage $[8,9]$. The pathophysiological development of secondary tissue damage in SCIs occurs through a complicated progression of cellular and molecular events [2]. Following the immediate mechanical damage to neural and other soft tissues, there is ischemic cellular death and edema, along with the infiltration of inflammatory cells, demyelination, and scarring and cyst formation at the lesion site. Because the injury compromises the blood-spinal cord barrier, immune cells migrate from the periphery and elicit an inflammatory response. The infiltration of neutrophils, macrophages, and lymphocytes helps fight infection and clear debris, but it also sets off the release of dozens of proinflammatory cytokines and chemokines that have been shown to negatively influence glial and neuronal cells. Another consequence is the production of reactive astrocytes, which in addition to producing inflammatory cytokines, ultimately participate in the formation of scar tissue. All of these processes eventually lead to loss of neuronal function.

Therapeutic emphasis in clinical and preclinical studies has been on cellular and molecular therapeutic interventions and includes transplantation or administration of a variety of cell types, neuroprotective agents, and growth factors $[8,10-12]$. But many are associated with several limitations, including the need for immunosuppressive drugs and a mode of continuous infusion $[13,14]$, which naturally raises the question of translation to patient care and how easily such a treatment could be administered to encumbered patients.

Keratin biomaterials have unique chemical, physical, and biological properties and have shown a significant ability for nerve repair in peripheral nerve injuries that surpasses conventional biomaterials; yet they do not rely on some of the more complex technologies needed to enhance other therapies, such as the addition of cells, growth factors, and/or gene constructs [13-18]. These studies using other biomaterial fillers such as hyaluronic acid, collagen, and fibrin gels and scaffolds, as well as numerous tubular devices made from synthetic and natural polymers, have shown promise, primarily in rodent studies, but relatively few have translated clinically and no filler materials are currently approved for human use by the US Food and Drug Administration. Studies using keratin biomaterial hydrogel fillers improved peripheral nerve regeneration by promoting Schwann cell activity via chemotaxis, increasing attachment and proliferation, and up-regulating genes that contribute to cell proliferation and differentiation [19]. However, later studies demonstrated only a modest increase in cellular infiltration into the conduit at early time points [20]. Pace et al. found that while keratin hydrogel fulfills primarily a structural role, increased myelin debris clearance in the distal nerve stump and reduced inflammatory cell influx also may influence the regenerative process in peripheral nerve regeneration.
Keratin biomaterials constructed from human hair are particularly versatile and can be processed into hydrogels, coatings, films, and sponges. The purpose of the current study was to investigate the potential use of a keratin hydrogel in SCI following a hemisection injury. Based on our earlier work in peripheral nerves, we postulated that keratin would be able to overcome the deterrent environment normally caused by SCI and provide a permissive milieu that supports repair and regeneration, as well as functional recovery. To investigate this, a rat hemisection model was used. This study addressed the critical question of assessing functional outcomes, which some previous studies have neglected. We hypothesized that treatment with a keratin hydrogel would encourage restoration of the ability to walk as well as void the bladder, the two most common disabilities present in persons with SCI.

\section{Materials and Methods}

2.1. Keratin Hydrogel Preparation. Isolation of the crude keratin material was achieved using previously described methods [19, 21]. Briefly, human hair was oxidized using peracetic acid followed by washing with deionized (DI) water to remove the residual oxidant. The soluble keratin proteins were extracted in tris(hydroxymethyl) aminomethane (Tris) base and DI water. The pooled solution was then dialyzed and neutralized ( $\mathrm{pH}$ 7.4) with $\mathrm{NaOH}$, lyophilized, and ground into powder. The keratin powder was sterilized using a $25 \mathrm{kGy}$ dose of $\gamma$-irradiation. To form hydrogels, sterile lyophilized keratin was aseptically reconstituted to $15 \%(\mathrm{w} / \mathrm{v})$ solution in phosphate buffered saline (PBS) and left to shake at $37^{\circ} \mathrm{C}$ overnight.

\subsection{Rat Spinal Cord Hemisection Injury. 25 male Lewis} rats were randomly divided into two treatment groups, keratin hydrogel and saline treatment. All animal procedures were approved by Wake Forest University Animal Care and Use Committee and conducted according to the National Institutes of Health (NIH) Guide for the Care and Use of Laboratory Animals. All procedures were performed under sterile conditions. Rats were anesthetized using isoflurane $(1.5 \%-2 \%)$ and oxygen. An $\sim 2 \mathrm{~cm}$ incision was made along the thoracic vertebral level. The laminae were resected and the dura mater was incised to expose the spinal cord. A left lateral hemisection at T8-T9 was made by creating a cut along the midline of the cord. Immediately after, $100 \mu \mathrm{L}$ of keratin hydrogel or saline was placed into the gap. Dura mater and skin were closed using 4-0 Vicryl suture (Ethicon). Buprenorphine $(0.01 \mathrm{mg} \cdot \mathrm{kg})$ was administered after closing and as needed for analgesia. Cefazolin $(250 \mathrm{mg} / \mathrm{kg})$ was administered after closing and prophylactically for 2 weeks. Rats were given lower abdominal and bladder massages 34 times daily to aid digestion and bladder voiding until they were able to void independently.

2.3. Cystometric Analysis and Bladder Weight. Cystometric studies were conducted at 3 weeks and 6 weeks after hemisection injury in awake, moving rats, as previously 
described [22-24]. Briefly, the implanted bladder catheter was attached to a two-way valve that was connected to a pressure transducer and infusion pump. An ETH 400 (CD Sciences) transducer amplifier and MacLab/8e (Analog Digital Instruments) were connected to the pressure transducer. Saline was introduced at $10 \mathrm{~mL} / \mathrm{h}$ and micturition volumes (MVs) measured using a force-displacement transducer. Analyses were conducted over a 25-minute time period. After cystometric analysis, animals were sacrificed and bladders immediately harvested and individually weighed.

2.4. Evaluation of Hind Limb Locomotor Function. Gait properties were evaluated using a treadmill and the TreadScan software system. Prior to the hemisection injury, rats were introduced to a treadmill to acclimate them and obtain normal baseline levels of gait parameters. It has been previously shown that increased exposure to the treadmill preceding injury can lead to adverse training effects [25], so animals were given minimal exposure. The recording and analysis procedure has been previously described [25]. Briefly, a highspeed digital camera recorded the rats from underneath as they walked across a clear treadmill. The TreadScan software identifies each footprint and analyzes it for numerous gait parameters. All analyses were done using data from the lower right leg (per lateral hemisection injury).

2.5. Histology/Tissue Preparation. Rats were sacrificed at 3 weeks and 6 weeks after hemisection injury. Animals were anesthetized with a ketamine/xylazine $(100 \mathrm{mg} / \mathrm{mL}$ and $20 \mathrm{mg} / \mathrm{mL}$, resp.) solution and transcardially perfused with $4 \%$ paraformaldehyde. Spinal cords were dissected, removed, and stored in $30 \%$ sucrose for 24 hours at $4^{\circ} \mathrm{C}$. Cords were then cut into $2 \mathrm{~cm}$ segments, embedded in O.C.T compound tissue freezing medium (Sakura), flash-frozen using liquid nitrogen, and stored at $-80^{\circ} \mathrm{C}$. Serial sections were cut longitudinally using a cryostat at $15 \mu \mathrm{m}$ thick. Masson's trichrome staining was done to elucidate scar formation and tissue organization. Images were captured using a Zeiss Axio Vert.A1 (Carl Zeiss Microscopy).

2.6. Statistical Analyses. Comparisons between treatment groups were analyzed for statistically significant differences using Prism v5.0 (GraphPad Software, Inc.). All data are reported as mean \pm standard deviation (SD). Survival curves were derived by the Kaplan-Meier method and compared by log-rank test. One-way analysis of variance (ANOVA) was used to determine significant differences between groups with $n \geq 3$ for bladder weights. Repeated measures ANOVA with Tukey's post hoc test was run on the baseline averages to assess any significant differences due to time or treatment in gait characteristics. $P<0.05$ was considered statistically significant.

\section{Results}

3.1. Effect of Keratin Treatment on Survival. Kaplan-Meier survival curves demonstrate that treatment with keratin

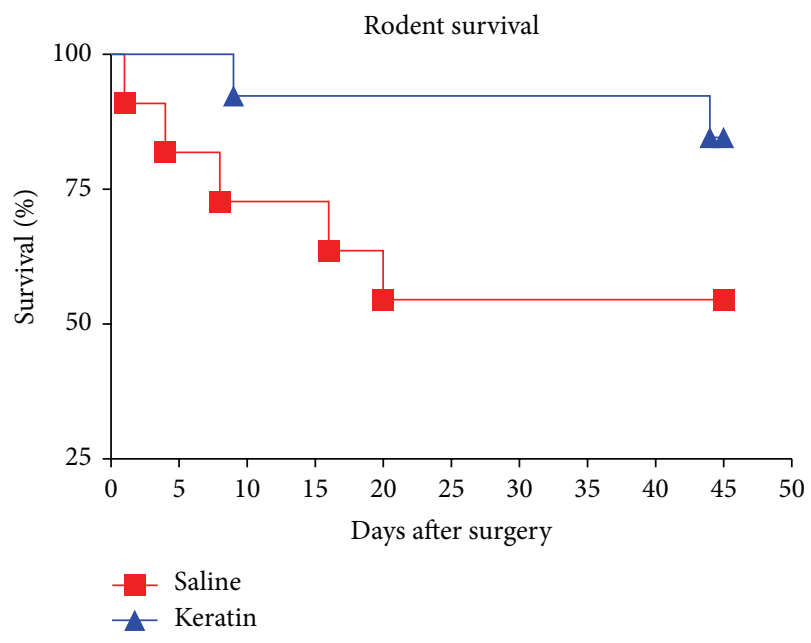

FIGURE 1: Kaplan-Meier survival curve of saline-treated rats $(n=11)$ compared to keratin- treated rats $(n=12)$.

hydrogel improved survival rates compared to saline treatment following hemisection injury (Figure 1). However, a high mortality rate in the saline group, particularly at long time points, contributed to the nonsignificance of this outcome.

3.2. Cystometry and Recovery of Bladder Function. Representative cystometrograms (CMGs) at 6 weeks after hemisection of the treatment groups and age-matched controls show distinct recovery of voiding abilities in rats that have been treated with keratin (Figure 2). Voiding pattern variability was observed within all groups at 3 weeks and 6 weeks, making a quantitative analysis difficult. However, trends in voiding pattern were observed. A typical saline-treated rat's bladder presented spontaneous uninhibited contractions not coupled with definite voids, which is indicative of an incontinent bladder (Figure 2(b)). Although the keratintreated rat's bladder has some instability during the filling phase (Figure 2(c)), it is much closer to a normal micturition pattern (Figure 2(a)), as it shows a regular pattern of definite voids coupled with pressure spikes and uninhibited contractions between voids (Figure 2(c)). This is representative of a functioning yet neurogenic bladder.

Average bladder weights at 6 weeks after hemisection demonstrate that the keratin group had significantly smaller bladders than the saline group overall (Figure 3). Bladders from the saline group were significantly larger than normal bladders from the uninjured age-matched control group, indicating a greater degree of hypertrophy. There is no significant difference between the keratin group and the uninjured age-matched controls.

3.3. Effects of Keratin Treatment on Hind Limb Motor Function. Keratin treatment was shown to significantly improve recovery of several gait properties (Figure 4). Stance time, which is the time elapsed while the foot is still in contact with the tread in its stance phase, was significantly higher in the keratin group at 3 weeks after hemisection (Figure 4(a)). 


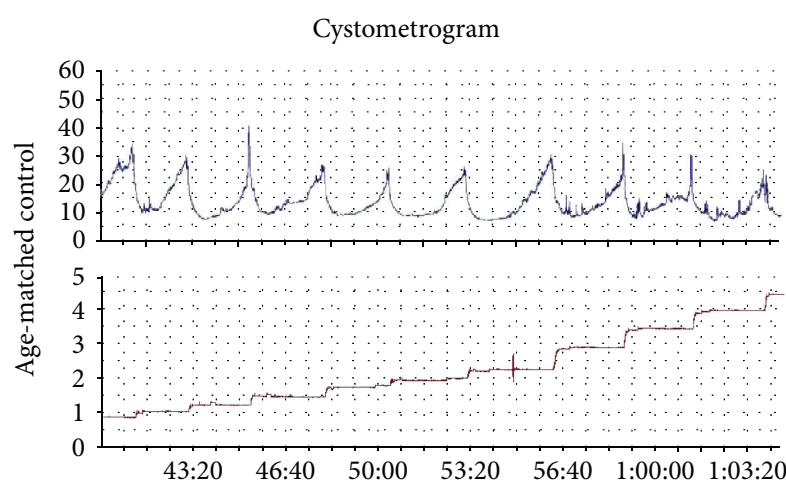

(a)

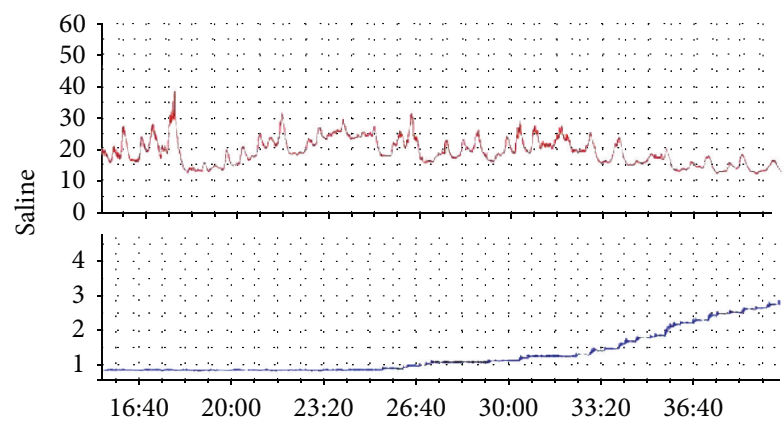

(b)

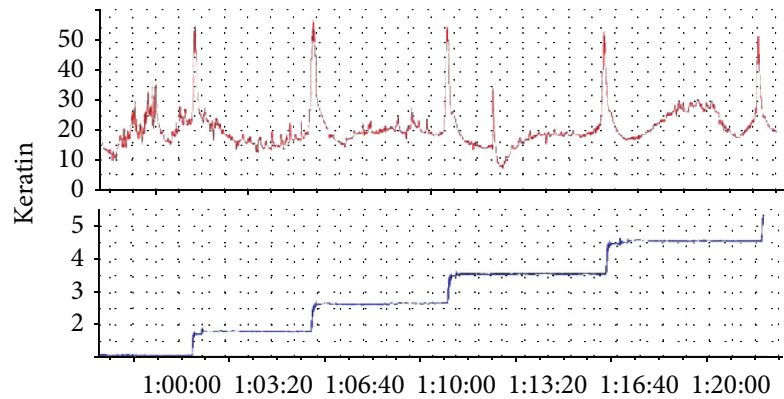

(c)

FIGURE 2: Representative cystometrograms showing the qualitative trends observed within each group. (a) Normal uninjured bladders show normal micturition pattern. (b) Saline-treated rats displayed uninhibited contractions not coupled with definite voids, while keratin-treated rats (c) showed a closer to normal voiding pattern. Each group includes a pressure curve $\left(0-60 \mathrm{~cm} / \mathrm{H}_{2} 0\right)$ versus a volume curve $(0-5 \mathrm{~mL})$ over a 25 -minute time scale ( $x$-axis).

Although the saline group showed increased recovery at 6 weeks, the keratin group still maintained its significance. Stride length, which is the distance the tread has moved while the foot is still in contact with it, also revealed a significant increase in the keratin-treated group by 6 weeks after hemisection (Figure 4(b)). Range of motion (ROM) or the distance between the farthest point and the closest point during each stride is typically a good indication of frontto-back motion. Again, by 6 weeks following hemisection injury, keratin treatment significantly improves recovery and is closer to the uninjured baseline level (Figure 4(c)).

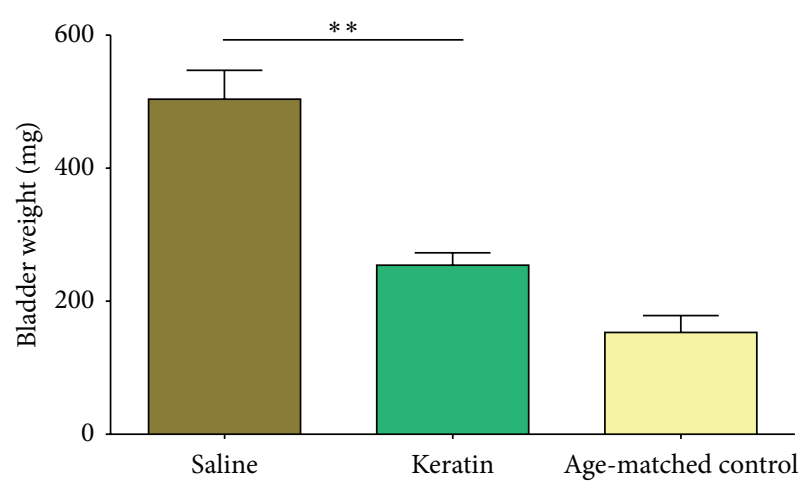

FIGURE 3: Average weight of bladders of animals treated with saline (control), keratin (experimental), and an uninjured age-matched control (positive control) at 6 weeks.

3.4. Histologic Analyses. By 3 weeks after hemisection, both keratin- and saline-treated rats show degeneration as well as scar formation at the lesion epicenter (Figure 5). However, by 6 weeks the keratin group displayed remarkable recovery to a near-normal anatomy. Of particular note is the lessening of the glial scar, where it is almost completely absent and limited to the lesioned half of the cord. Spinal cords from the salinetreated group had especially severe degeneration at 6 weeks after hemisection.

\section{Discussion/Conclusion}

SCIs are particularly difficult to treat and there are many experimental approaches being pursued, including the use of stem cells, small molecule therapies, and various autologous cell and tissue grafts. While some promising results have been noted, a recent stem cell clinical trial by Geron Corporation was halted, indicating that a clinical treatment using these complex approaches may still be many years away. This study has taken a simpler, biomaterials-based approach using a keratin hydrogel. Keratin biomaterials have previously demonstrated efficacy in promoting repair following injury in a peripheral nerve model in mouse, rat, rabbit, and, most recently, nonhuman primate models [19, 20, 26, 27]. Given the significance of those results, keratin was considered a viable candidate for use in the central nervous system. Moreover, hydrogels are especially well suited for SCIs because of intrinsic properties such as softness, highly porous three-dimensional structure, mimicry of the extracellular matrix (ECM), and a reduced foreign-body response [28]. In addition to encompassing these classic characteristics, studies have shown that keratin hydrogels are able to avoid any major fibrous capsule response, can support cellular and vascular infiltration, can evade foreign-body giant cell formation and graft rejection, and, because of the absence of genetic elements, is nonantigenic and nonimmunogenic $[21,29,30]$.

The purpose of this study was to investigate keratin's potential role in promoting repair following SCI. Due to the exploratory nature of this pilot study and the high mortality rate, the experiments were underpowered. Survival rates for 

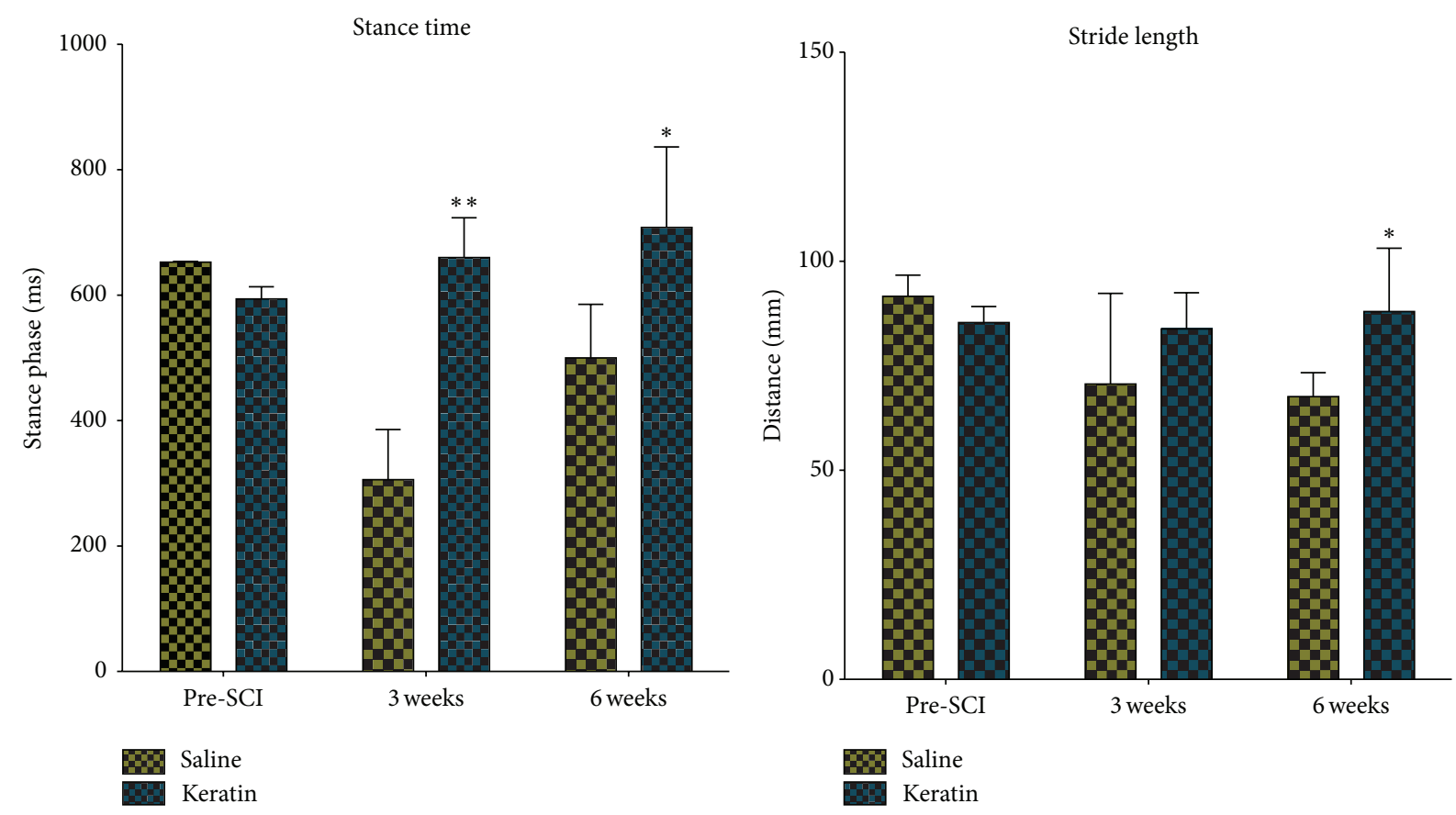

(a)

(b)

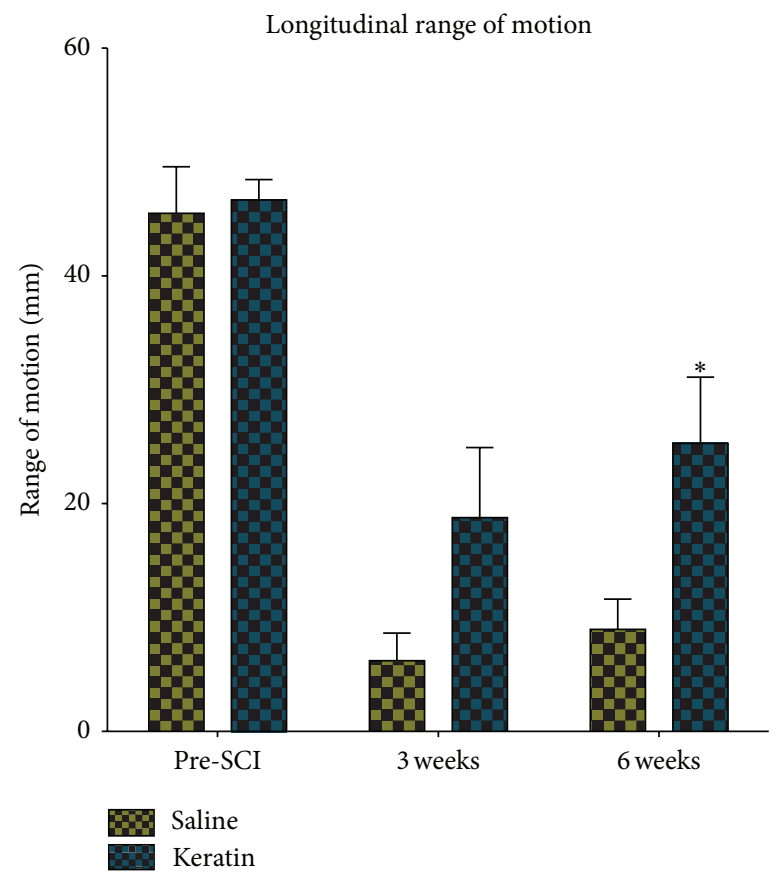

(c)

FIGURE 4: Gait analysis shows greater recovery of stance time (a) with keratin treatment at both 3 weeks and 6 weeks after injury. Keratintreated rats also showed significantly greater stride length (b) and longitudinal range of motion (c) by 6 weeks.

those rats treated with keratin were markedly greater than those treated with saline (Figure 1), but due to the lack of surviving animals in general, and in the 6 week saline group in particular, statistical significance in this particular outcome was not achieved. The attrition rates observed in this study were higher than expected, although this type of data is typically not published and difficult to obtain. Nevertheless, all animals were given the exact same postoperative care and treatment, so within the present study it is difficult to ignore the effects observed.

It is well known in SCI that urinary bladder control is one of the first problems patients encounter. The normal expectation after spinal cord damage above the level of S2 is an initial spinal shock where there is complete urinary retention with possible overflow incontinence, followed within 2-3 weeks by the initiation of an autonomic bladder, or 

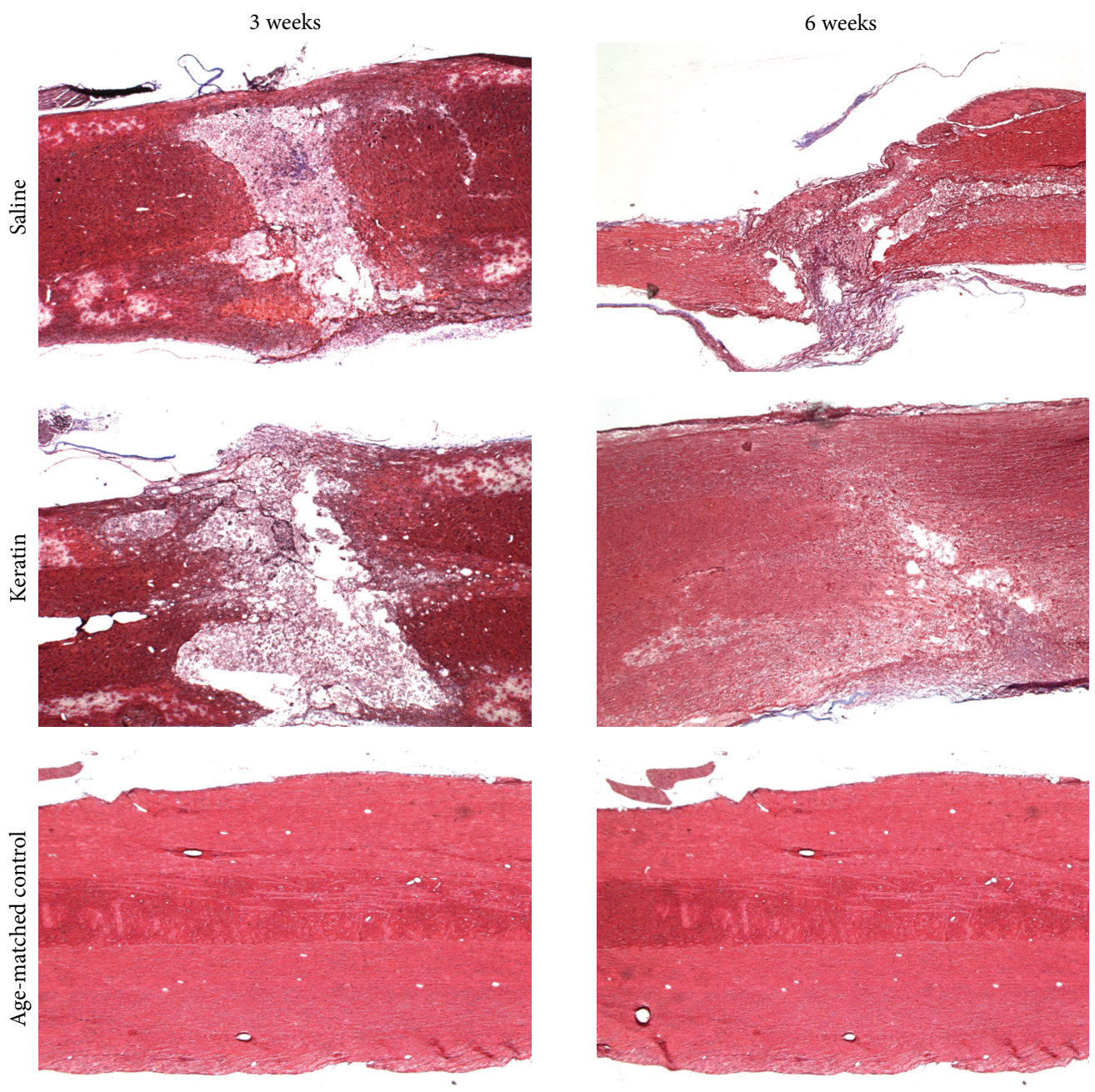

FIGURE 5: Trichrome staining of recovered spinal cords showed notable restoration of tissue in the keratin-treated group compared to saline by 6 weeks. While some scarring is evident, the deterioration that occurred with saline treatment was not evident in keratin-treated animals.

loss of micturition reflexes, abnormal amounts of residual urine, and involuntary urination. In the present study, the effect of keratin on regeneration in the spinal cord was studied and consequently bladder function was used as an indication of successful recovery. Our postulate was that, in rats, more rapid recovery from the spinal shock phase would be observed, and thus our analysis was focused on the 3week time point. As expected, the saline group showed severe nerve damage with no clear voiding pattern in any of the animals tested (Figure 2(b)). Pressure data showed marked uninhibited contractions and irregular voiding pattern that was not correlated with specific spikes in pressure. This pattern indicates loss of upper motor neuron control on the bladder and failure of nerve regeneration at the spinal cord level. In the keratin group, however, there was a more robust pattern of voiding in some of the animals tested and there were regular bouts of micturition in $60 \%$ (3/5) of animals tested (Figure 2(c)). The bladder pressure data showed a clear trend toward normalization of micturition, albeit with a sustained increase in uninhibited contractions in between those sections. These data suggest at least some regeneration at the spinal cord level, allowing at least minor upper center control of the bladder to resume.

Another indication of a neurogenic response by the bladder to SCI is the degree of hypertrophy. As a muscle, the bladder will compensate for any voiding problems by enlarging. Measuring the weights of bladders taken from each treatment group provides an indication of how the bladder is responding. Keratin treatment yielded significantly smaller bladders compared to saline treatment and there was no significant difference between uninjured age-matched controls and the keratin treatment group bladder weights (Figure 3).

Another substantial functional outcome following SCI is gait analysis. The ability to walk is a good assessment of SCI deficit because it shows definitively that neurite connections have regenerated to an extent of becoming functional. Moreover, walking is noted to be the highest priority for SCI patients [31, 32]. A thoracic hemisection injury, such as the one employed for this study, will cause the animal to lose the use of one hind limb, in this case the right hind limb. The TreadScan system allows the animal to 
be recorded from underneath a clear treadmill as they are walking. It provides a highly sensitive and objective output that in most, but not all, cases is more insightful than the classic open-field assessments [25]. Baseline readings taken at day 0 are pre-SCI and show how a normal response appears (Figure 4). Keratin treatment promoted a significant recovery of all three properties by 6 weeks. Previous studies using mouse models of SCI have found that injured animals display a phase of initial recovery, which then plateaus by 2-3 weeks [33-35]. This is consistent with trends observed within the saline treatment group, as there was no significant difference between 3 weeks and 6 weeks for all three gait properties measured. In contrast, there was significant restoration of stance time, stride length, and longitudinal ROM to nearnormal levels with keratin treatment, thus showing recovery occurring longer and later.

Regeneration of neurons is limited by many factors that contribute to an environment that is impermissible to growth and repair, unlike regeneration of peripheral nerves which can occur at a rate of up to $5 \mathrm{~mm}$ per day [36]. Previous studies have shown that spinal cord regeneration is possible if the lesion milieu promotes and supports growth [36-38]. Specifically, the inhibitory lesion site or glial scar must be prevented or kept at a minimum to allow CNS axons to grow [36, 38-41]. Spared white matter has been shown to correlate well with functional recovery following SCI [34, 35, 42, 43]. This study demonstrated consistent findings in this regard, as seen at 6 weeks after hemisection where the keratin treatment group spinal cords showed greater remodeling (Figure 5), which is also the time point at which the greatest functional recovery appeared to take place (Figures 2 and 4). At 3 weeks after hemisection, the keratin treatment group's spinal cords were not visibly different from the saline treatment group, as both displayed degeneration and some scar tissue formation. By 6 weeks after hemisection, it is apparent how similar the tissue structure and organization of the keratin-treated cord are to the uninjured spinal cord (Figure 5). There are several potential cellular and molecular mechanisms that could be responsible for regeneration at this level, such as modulation of cell-mediated inflammatory cascades and/or promotion of glial cell remodeling activity. However, previous studies would suggest that keratin is not acting directly on neurons or axons but rather as a structural support, providing an environment more conducive to growth, which is achieved in the spinal cord through promoting beneficial cell types like oligodendrocytes, microglia, and macrophages.

\section{Conclusion}

This pilot study suggests the potential of a keratin biomaterial-based treatment in SCI applications. Although the study was underpowered, gait parameters did reach statistical significance and other notable observations were made. In general, keratin treatment seemed to improve survival rates compared to saline treatment. Rats treated with keratin also appeared to have greater recovery of bladder function. Although micturition patterns are shown in qualitative terms, the average weight of bladders was significantly smaller with keratin treatment, indicating less hypertrophy and thus restored innervation of the bladder muscle. There was also a significant improvement in hind limb motor function recovery with keratin treatment. Histological analysis suggests an increased cellular presence with keratin, which may be indicative of several potential mechanisms of action. However, further studies are needed to confirm these findings in a more statistically robust design as well as explore these initial observations and address mechanistic questions.

\section{Conflict of Interests}

Author Mark E. Van Dyke holds stock and is an officer in the company KeraNetics, LLC, that provided partial funding for this research. Wake Forest School of Medicine has a potential financial interest in KeraNetics through licensing agreements.

\section{Acknowledgments}

The authors would like to thank Clever Sys, Inc., for the generous loan of the treadmill and TreadScan software, Lauren Pace for her assistance in animal husbandry, and David Burmeister for his assistance in the cystometric analysis. Funding was provided by KeraNetics, LLC, and the Errett Fisher Foundation.

\section{References}

[1] National Spinal Cord Injury Statistical Center, Facts and Figures at a Glance, University of Alabama at Birmingham, Birmingham, Ala, USA, 2013.

[2] A. Blesch and M. H. Tuszynski, "Spinal cord injury: plasticity, regeneration and the challenge of translational drug development," Trends in Neurosciences, vol. 32, no. 1, pp. 41-47, 2009.

[3] M. G. Fehlings, "Editorial: recommendations regarding the use of methylprednisolone in acute spinal cord injury: making sense out of the controversy," Spine, vol. 26, supplement 24, pp. S56S57, 2001.

[4] S. J. Gerndt, J. L. Rodriguez, J. W. Pawlik et al., "Consequences of high-dose steroid therapy for acute spinal cord injury," The Journal of Trauma, vol. 42, no. 2, pp. 279-284, 1997.

[5] R. J. Hurlbert and M. G. Hamilton, "Methylprednisolone for acute spinal cord injury: 5-year practice reversal," The Canadian Journal of Neurological Sciences, vol. 35, no. 1, pp. 41-45, 2008.

[6] A. M. Bagnall, L. Jones, S. Duffy, and R. P. Riemsma, "Spinal fixation surgery for acute traumatic spinal cord injury," The Cochrane Database of Systematic Reviews, no. 1, Article ID CD004725, 2008.

[7] M. G. Fehlings and R. G. Perrin, "The role and timing of early decompression for cervical spinal cord injury: update with a review of recent clinical evidence," Injury, vol. 36, no. 2, supplement, pp. S13-S26, 2005.

[8] P. Gál, P. Kravcuková, M. Mokrý, and D. Kluchová, "Chemokines as possible targets in modulation of the secondary damage after acute spinal cord injury: a review," Cellular and Molecular Neurobiology, vol. 29, no. 6-7, pp. 1025-1035, 2009. 
[9] N. Olby, "Current concepts in the management of acute spinal cord injury," Journal of Veterinary Internal Medicine, vol. 13, no. 5, pp. 399-407, 1999.

[10] G. Cantarella, G. di Benedetto, M. Scollo et al., "Neutralization of tumor necrosis factor-related apoptosis-inducing ligand reduces spinal cord injury damage in mice," Neuropsychopharmacology, vol. 10, no. 4, pp. 1-13, 2010.

[11] D. Gupta, C. H. Tator, and M. S. Shoichet, "Fast-gelling injectable blend of hyaluronan and methylcellulose for intrathecal, localized delivery to the injured spinal cord," Biomaterials, vol. 27, no. 11, pp. 2370-2379, 2006.

[12] S. Woerly, V. D. Doan, N. Sosa, J. de Vellis, and A. EspinosaJeffrey, "Prevention of gliotic scar formation by NeuroGel allows partial endogenous repair of transected cat spinal cord," Journal of Neuroscience Research, vol. 75, no. 2, pp. 262-272, 2004.

[13] D. D. Pearse, F. C. Pereira, A. E. Marcillo et al., "cAMP and Schwann cells promote axonal growth and functional recovery after spinal cord injury," Nature Medicine, vol. 10, no. 6, pp. 610616, 2004.

[14] S. Thuret, L. D. Moon, and F. H. Gage, "Therapeutic interventions after spinal cord injury," Nature Reviews Neuroscience, vol. 7, no. 8, pp. 628-643, 2006.

[15] F. Z. Cui, W. M. Tian, S. P. Hou, Q. Y. Xu, and I.-S. Lee, "Hyaluronic acid hydrogel immobilized with RGD peptides for brain tissue engineering," Journal of Materials Science: Materials in Medicine, vol. 17, no. 12, pp. 1393-1401, 2006.

[16] J. Park, E. Lim, S. Back, H. Na, Y. Park, and K. Sun, "Nerve regeneration following spinal cord injury using matrix metalloproteinase-sensitive, hyaluronic acid-based biomimetic hydrogel scaffold containing brain-derived neurotrophic factor," Journal of Biomedical Materials Research A, vol. 93, no. 3, pp. 1091-1099, 2010.

[17] G. A. Silva, C. Czeisler, K. L. Niece et al., "Selective differentiation of neural progenitor cells by high-epitope density nanofibers," Science, vol. 303, no. 5662, pp. 1352-1355, 2004.

[18] V. M. Tysseling-Mattiace, V. Sahni, K. L. Niece et al., "Selfassembling nanofibers inhibit glial scar formation and promote axon elongation after spinal cord injury," The Journal of Neuroscience, vol. 28, no. 14, pp. 3814-3823, 2008.

[19] P. Sierpinski, J. Garrett, J. Ma et al., "The use of keratin biomaterials derived from human hair for the promotion of rapid regeneration of peripheral nerves," Biomaterials, vol. 29, no. 1, pp. 118-128, 2008.

[20] L. A. Pace, J. F. Plate, T. L. Smith, and M. E. van Dyke, "The effect of human hair keratin hydrogel on early cellular response to sciatic nerve injury in a rat model," Biomaterials, vol. 34 , no. 24, pp. 5907-5914, 2013.

[21] R. C. de Guzman, M. R. Merrill, J. R. Richter, R. I. Hamzi, O. K. Greengauz-Roberts, and M. E. van Dyke, "Mechanical and biological properties of keratose biomaterials," Biomaterials, vol. 32, no. 32, pp. 8205-8217, 2011.

[22] D. Burmeister, T. Aboushwareb, J. Tan, K. Link, K.-E. Andersson, and G. Christ, "Early stages of in situ bladder regeneration in a rodent model," Tissue Engineering A, vol. 16, no. 8, pp. 25412551, 2010.

[23] T. C. Chai, H. Gemalmaz, K.-E. Andersson, J. B. Tuttle, and W. D. Steers, "Persistently increased voiding frequency despite relief of bladder outlet obstruction," The Journal of Urology, vol. 161, no. 5, pp. 1689-1693, 1999.

[24] A. Malmgren, C. Sjögren, B. Uvelius et al., "Cystometrical evaluation of bladder instability in rats with infravesical outflow obstruction," The Journal of Urology, vol. 137, no. 6, pp. 12911294, 1987.

[25] J. E. Beare, J. R. Morehouse, W. H. DeVries et al., "Gait analysis in normal and spinal contused mice using the treadscan system," Journal of Neurotrauma, vol. 26, no. 11, pp. 2045-2056, 2009.

[26] P. J. Apel, J. P. Garrett, P. Sierpinski et al., "Peripheral nerve regeneration using a keratin-based scaffold: long-term functional and histological outcomes in a mouse model," The Journal of Hand Surgery, vol. 33, no. 9, pp. 1541-1547, 2008.

[27] P. S. Hill, P. J. Apel, J. Barnwell et al., "Repair of peripheral nerve defects in rabbits using keratin hydrogel scaffolds," Tissue Engineering A, vol. 17, no. 11-12, pp. 1499-1505, 2011.

[28] K. S. Straley, C. W. Foo, and S. C. Heilshorn, "Biomaterial design strategies for the treatment of spinal cord injuries," Journal of Neurotrauma, vol. 27, no. 1, pp. 1-19, 2010.

[29] R. C. de Guzman, J. M. Saul, M. D. Ellenburg et al., "Bone regeneration with BMP-2 delivered from keratose scaffolds," Biomaterials, vol. 34, no. 6, pp. 1644-1656, 2013.

[30] J. Kirfel, T. M. Magin, and J. Reichelt, "Keratins: a structural scaffold with emerging functions," Cellular and Molecular Life Sciences, vol. 60, no. 1, pp. 56-71, 2003.

[31] P. L. Ditunno, M. Patrick, M. Stineman, and J. F. Ditunno, "Who wants to walk? Preferences for recovery after SCI: a longitudinal and cross-sectional study," Spinal Cord, vol. 46, no. 7, pp. 500506, 2008.

[32] P. L. Ditunno, M. Patrick, M. Stineman, B. Morganti, A. F. Townson, and J. F. Ditunno, "Cross-cultural differences in preference for recovery of mobility among spinal cord injury rehabilitation professionals," Spinal Cord, vol. 44, no. 9, pp. 567575, 2006.

[33] D. M. Basso, L. C. Fisher, A. J. Anderson, L. B. Jakeman, D. M. McTigue, and P. G. Popovich, "Basso mouse scale for locomotion detects differences in recovery after spinal cord injury in five common mouse strains," Journal of Neurotrauma, vol. 23, no. 5, pp. 635-659, 2006.

[34] P. L. Kuhn and J. R. Wrathall, "A mouse model of graded contusive spinal cord injury," Journal of Neurotrauma, vol. 15, no. 2, pp. 125-140, 1998.

[35] M. Ma, D. M. Basso, P. Walters, B. T. Stokes, and L. B. Jakeman, "Behavioral and histological outcomes following graded spinal cord contusion injury in the $\mathrm{C} 57 \mathrm{Bl} / 6$ mouse," Experimental Neurology, vol. 169, no. 2, pp. 239-254, 2001.

[36] J. B. Recknor and S. K. Mallapragada, "Nerve regeneration: tissue engineering strategies," in The Biomedical Engineering Handbook: Tissue Engineering and Artificial Organs, J. D. Bronzino, Ed., pp. 48-41, Taylor \& Francis, Boca Raton, Fla, USA, 3rd edition, 2006.

[37] E. J. Bradbury and S. B. McMahon, "Spinal cord repair strategies: why do they work?” Nature Reviews Neuroscience, vol. 7, no. 8, pp. 644-653, 2006.

[38] G. Yiu and Z. He, "Glial inhibition of CNS axon regeneration," Nature Reviews Neuroscience, vol. 7, no. 8, pp. 617-627, 2006.

[39] T. Karnezis, W. Mandemakers, J. L. McQualter et al., “The neurite outgrowth inhibitor Nogo A is involved in autoimmunemediated demyelination," Nature Neuroscience, vol. 7, no. 7, pp. 736-744, 2004.

[40] H. Wang, Y. Katagiri, T. E. McCann et al., "Chondroitin-4sulfation negatively regulates axonal guidance and growth," Journal of Cell Science, vol. 121, part 18, pp. 3083-3091, 2008.

[41] H. Zhang, K. Uchimura, and K. Kadomatsu, "Brain keratan sulfate and glial scar formation," Annals of the New York Academy of Sciences, vol. 1086, pp. 81-90, 2006. 
[42] Q. Cao, Y. P. Zhang, C. Iannotti et al., "Functional and electrophysiological changes after graded traumatic spinal cord injury in adult rat," Experimental Neurology, vol. 191, supplement 1, pp. S3-S16, 2005.

[43] Y. Li, R. J. Oskouian, Y.-J. Day, J. A. Kern, and J. Linden, "Optimization of a mouse locomotor rating system to evaluate compression-induced spinal cord injury: correlation of locomotor and morphological injury indices," Journal of Neurosurgery: Spine, vol. 4, no. 2, pp. 165-173, 2006. 

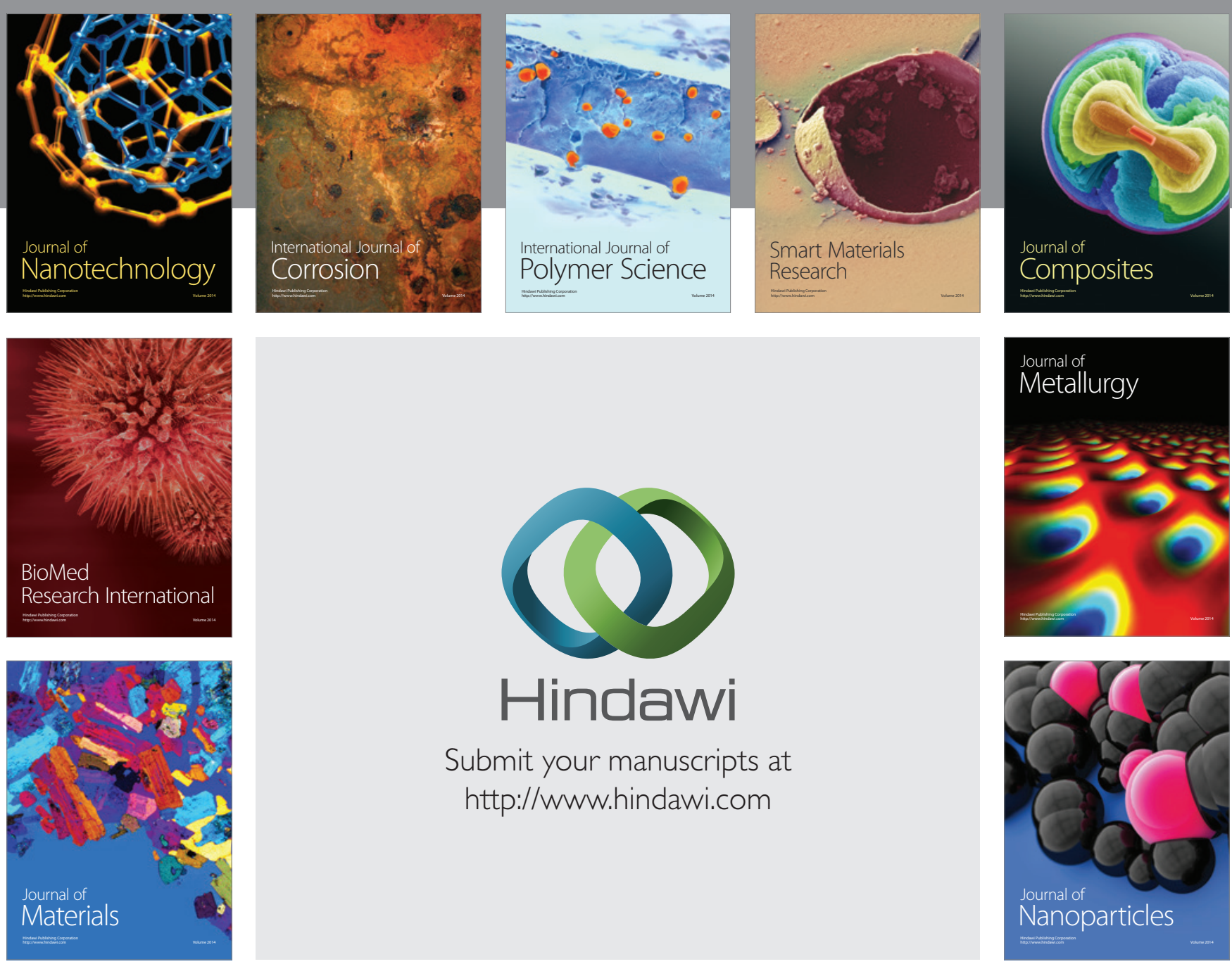

Submit your manuscripts at http://www.hindawi.com
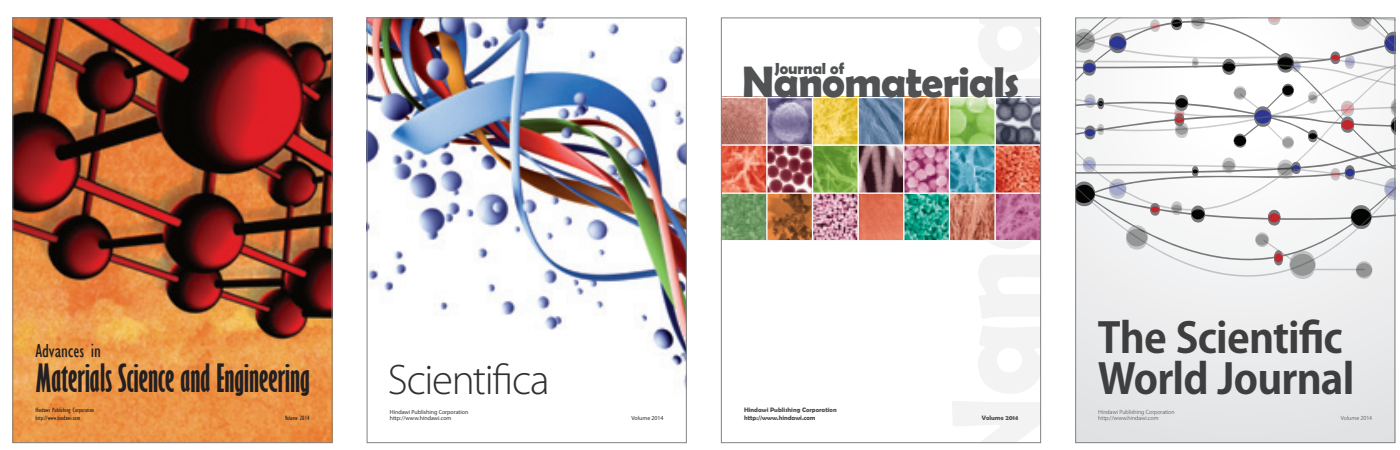

\section{The Scientific World Journal}
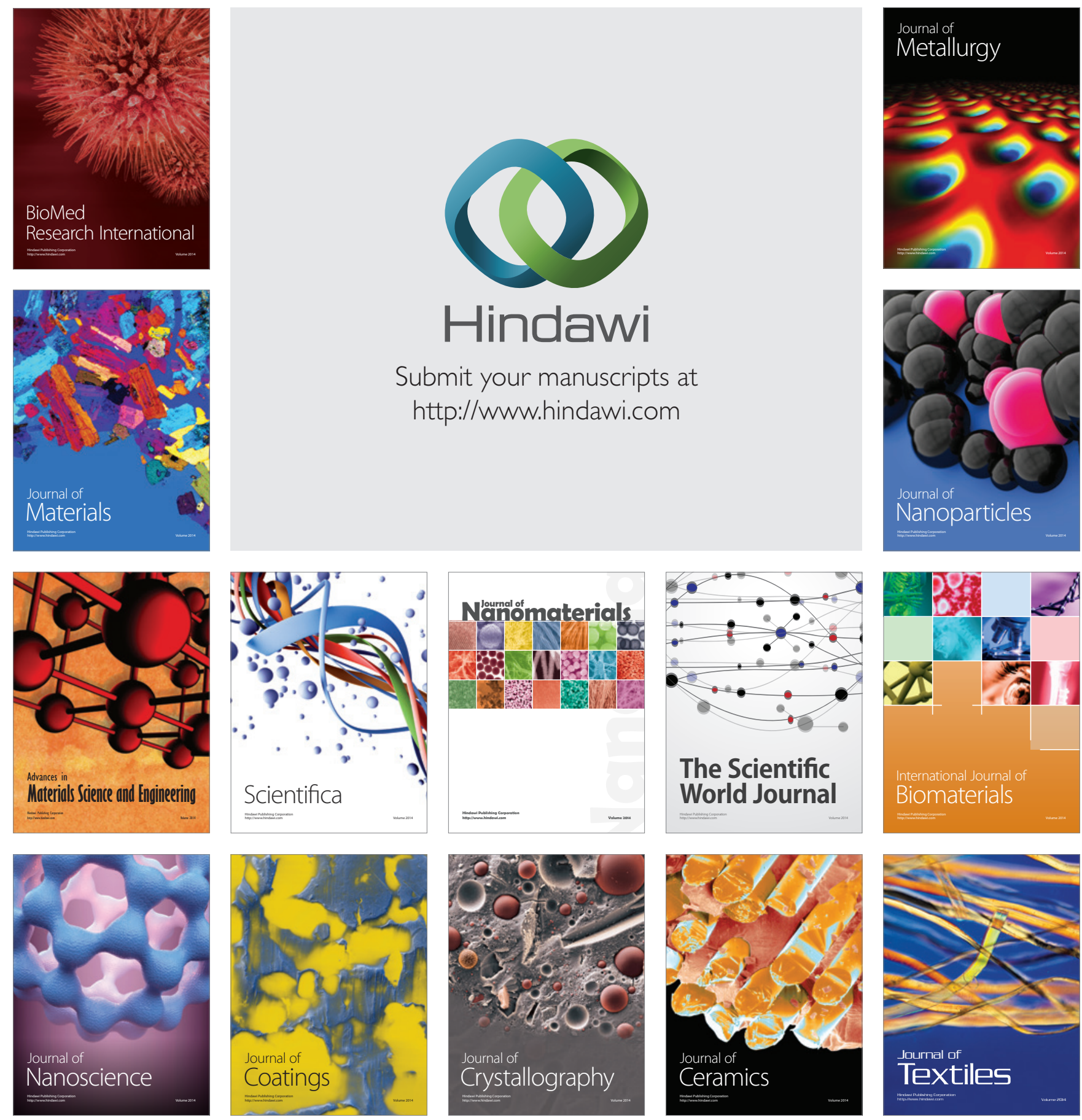